\title{
Trade-Off in the Sound Localization Abilities of Early Blind Individuals between the Horizontal and Vertical Planes
}

\author{
Patrice Voss, ${ }^{1,2}$ Vanessa Tabry, ${ }^{3}$ and Robert J. Zatorre ${ }^{1,2}$ \\ ${ }^{1}$ Montreal Neurological Institute, McGill University, Montréal, Québec H3A 2B4, Canada, ${ }^{2}$ International Laboratory for Brain, Music and Sound Research \\ (BRAMS), Montréal, Québec H2V 4P3, Canada, and ³Department of Psychology, Concordia University, Montréal, Québec H3A 2B4, Canada
}

\begin{abstract}
There is substantial evidence that sensory deprivation leads to important cross-modal brain reorganization that is paralleled by enhanced perceptual abilities. However, it remains unclear how widespread these enhancements are, and whether they are intercorrelated or arise at the expense of other perceptual abilities. One specific area where such a trade-off might arise is that of spatial hearing, where blind individuals have been shown to possess superior monaural localization abilities in the horizontal plane, but inferior localization abilities in the vertical plane. While both of these tasks likely involve the use of monaural cues due to the absence of any relevant binaural signal, there is currently no proper explanation for this discrepancy, nor has any study investigated both sets of abilities in the same sample of blind individuals. Here, we assess whether the enhancements observed in the horizontal plane are related to the deficits observed in the vertical plane by testing sound localization in both planes in groups of blind and sighted persons. Our results show that the blind individuals who displayed the highest accuracy at localizing sounds monaurally in the horizontal plane are also the ones who exhibited the greater deficit when localizing in the vertical plane. These findings appear to argue against the idea of generalized perceptual enhancements in the early blind, and instead suggest the possibility of a trade-off in the localization proficiency between the two auditory spatial planes, such that learning to use monaural cues for the horizontal plane comes at the expense of using those cues to localize in the vertical plane.
\end{abstract}

Key words: azimuth; blindness; sound localization; vertical

\section{Introduction}

Previous work has shown that some early blind (EB) individuals (approximately half of those tested) can localize sound sources in the horizontal plane with a high degree of accuracy when having to do so with one ear occluded (Lessard et al., 1998; Gougoux et al., 2005). While several auditory cues play a role in localizing monaurally in the horizontal plane, including head shadow cues (Van Wanrooij and Van Opstal, 2004), subsequent findings have strongly suggested that it is specifically pinna spectral cues that play a key role in the monaural localization abilities of those EB persons who show the enhancement. For instance, Doucet et al. (2005) showed that the supranormal monaural localization performance of EB persons was significantly decreased by the occlusion of the pinna. Subsequently, Voss et al. (2011) showed that

Received Nov. 3, 2014; revised Feb. 17, 2015; accepted March 4, 2015.

Author contributions: P.V. and R.J.Z. designed research; P.V. and V.T. performed research; P.V., V.T., and R.J.Z. analyzed data; P.V. and R.J.Z. wrote the paper.

This research was funded by the Canadian Institutes of Health Research, the Canada Fund for Innovation, and the Fonds Québécois de Recherche Nature et Technologies/Société et Culture via its funding of the Centre for Research in Brain, Language and Music. P.V. was supported by a Banting Postdoctoral Fellowship, which is administered by the Natural Science and Engineering Research Council of Canada. We thank all of the individuals who volunteered to participate in this study as well as the Institut Nazareh et Louis-Braille for its assistance in recruiting blind participants. We also thank Marc Schönwiesner and Régis Trapeau for their assistance in programming the tasks and setting up the equipment

The authors declare no competing financial interests.

Correspondence should be addressed to Patrice Voss, Department of Neurology and Neurosurgery, Montreal Neurological Institute, 3801 Rue University, Montréal, QC H3A 2B4, Canada. E-mail: patrice.voss@mcgill.ca.

DOI:10.1523/JNEUROSCI.4544-14.2015

Copyright $\odot 2015$ the authors $\quad 0270-6474 / 15 / 356051-06 \$ 15.00 / 0$ when having to discriminate between sounds with spectral profiles that simulated different spatial positions based on headrelated transfer functions (HRTFs; i.e., spectral cues), the task was best performed by those EB persons displaying superior monaural sound localization abilities. Furthermore, the EB have been shown to have better sound source discrimination abilities in peripheral auditory space near the interaural axis (Röder et al., 1999; Voss et al., 2004), where binaural cues are insufficient to resolve whether a sound source lies in front or behind the axis and where spectral cues can help to resolve this ambiguity. The abovehighlighted evidence therefore leads to the hypothesis that the superior ability of some early blind individuals to localize sounds more accurately rests on their ability to make better use of spectral cues.

However, this spectral cue hypothesis is challenged by studies showing that the EB are worse than sighted individuals in localizing sound targets in the vertical mid-sagittal plane (Zwiers et al., 2001; Lewald, 2002), an ability that is also believed to rest primarily on the use of spectral cues (Middlebrooks and Green, 1991; Blauert, 1997). Although to date no study has investigated both sets of abilities in the same sample of EB persons, nor is there currently a proper explanation for this discrepancy, the aforementioned studies, taken together, suggest the possible existence of a trade-off in the localization proficiency between the two auditory spatial planes. This also suggests that the relevant spectral cues for accurate localization in both planes might differ in their nature, which could explain the drop-off in performance in the vertical plane observed in the early blind. 
Therefore, to investigate the potential existence of trade-offs in the development of enhanced perceptual abilities in the EB, and to address the long-standing discrepancy regarding their spatial hearing abilities, we asked both EB and sighted individuals to perform sound localization tasks under the following three distinct listening conditions: binaural localization in the horizontal plane; monaural localization (with one ear occluded) in the horizontal plane; and binaural localization in the vertical plane. EB subjects were divided into two groups, in accordance with previous work showing a clear dichotomy in their performance on the monaural horizontal localization task, where approximately half of them can accurately localize sounds from the side of the occluded ear, and the other half are rather poor at localizing from the occluded side, similar to sighted individuals (Lessard et al., 1998; Doucet et al., 2005; Gougoux et al., 2005). Neuroimaging findings also support this division as it is only those who show superior accuracy that also display cross-modal recruitment of their visual cortices (Gougoux et al., 2005; Voss et al., 2008, 2011).

\section{Materials and Methods}

Subjects. Eleven EB subjects (three females; mean age, $34.5 \pm 12.11$ years) and twelve sighted subjects (three female; mean age, $35.7 \pm 12.87$ years) took part in this study. The visual deficit of each EB resulted from anomalies in peripheral structures with no neurological deficits, and led to total blindness except for minimal residual light sensitivity in one subject. The average age of blindness onset was $0.6 \pm 1.2$ years. Audiometric thresholds were assessed for all participants, and indicated normal and comparable hearing in both ears (hearing thresholds $\leq 20 \mathrm{~dB}$ hearing level at frequencies between 0.25 and $8 \mathrm{kHz}$ ), and were comparable across groups. All subjects gave written informed consent in accordance with the guidelines approved by the Montreal Neurological Institute ethics board and the Centre de Recherche Interdisciplinaire en Réadaptation, which coordinates research with blind subjects sponsored by the Nazareth and Louis-Braille Institute for the blind.

Conditions. All subjects were required to localize sounds in the following three separate experimental conditions: (1) binaural sound localization in the horizontal plane; (2) monaural localization (with one ear occluded) in the horizontal plane; and (3) binaural localization in the vertical plane. While all subjects were blindfolded for the duration of each condition, the blindfold was removed between conditions to avoid any potential enhancements associated with short-term visual deprivation in the sighted subjects (Lewald, 2007). In monaural conditions, one ear was obstructed with a soft foam earplug (mean attenuation, $37.5 \mathrm{~dB}$ SPL) and was covered by a hearing protector muff (mean attenuation, 29 dB SPL). Note that while the chosen ear for occlusion was counterbalanced across subjects, the behavioral results were transformed such that the left side was arbitrarily assigned to correspond to the obstructed ear. Thus, in the behavioral data presented for the monaural task in the horizontal plane, negative angles on the abscissa correspond to the obstructed side, while positive angles correspond to the unobstructed side.

Apparatus. The experiment was performed in a hemi-anechoic chamber $(2.5 \times 5.5 \times 2.5 \mathrm{~m})$. The acoustic apparatus used to test sound localization consisted of 25 loudspeakers on the horizontal plane and 18 on the vertical plane, mounted on two semicircular railings with a radius of $90 \mathrm{~cm}$. Each location was sampled five times in each condition. The positions of the loudspeakers ranged from -90 to $+90^{\circ}$ on the horizontal plane, and from -45 to $+82.5^{\circ}$ on the vertical plane, thus providing a spatial resolution of $7.5^{\circ}$ in both planes. Subjects were seated such that the speakers in the horizontal plane were positioned at ear level and those in the vertical plane were aligned with the subjects' mid-sagittal plane $\left(0^{\circ}\right.$ azimuth, $0^{\circ}$ elevation). Two fabric rulers were put in place along the semicircular railings; this was done so that an experimenter present could note laser-pointed locations (see Procedure).

Stimuli. Sound localization tests were controlled by a custom-designed Matlab script (r.2009a; MathWorks), and stimuli were generated using TDT System 3 (Tucker-Davis Technology). The stimuli consisted of 100 ms pink noise bursts (10 ms rise/fall times) presented at a $50 \mathrm{~dB}$ SPL, as measured at the head position of the subjects.

Procedure. Subjects were seated in a fixed chair in front of the two semicircular railings and were required to indicate the location of short noise bursts delivered through a randomly selected loudspeaker (in a predetermined auditory spatial plane that was known to the subjects) with a hand-held laser pointer in their dominant hand (all subjects were right handed). This hand-pointing method was recently shown (Tabry et al., 2013) to be the most accurate when sighted subjects have to localize sounds in both the horizontal and vertical planes while blindfolded. The location indicated by the subject was marked down by an experimenter present in the room. Subjects were also instructed to maintain a head position pointing straight ahead $\left(0^{\circ}\right.$ azimuth, $0^{\circ}$ elevation $)$ until the end of the stimulus presentation, and were required to return to that position before starting the next trial. Failure to do so would result in the inability to start the next trial, as subjects wore an elastic cap with a magnetic receiver of a 3D digitizer system (ISOTRAK II, Polhemus) that recorded the head position and did not allow the trials to continue until the subjects had properly returned to the center position. When the head was properly positioned, a brief high-frequency tone was played via the speaker directly above the head, and was followed by the sound burst to be localized. An improper head position produced a lower-frequency tone, and the subject was required to reposition their head appropriately.

Before beginning the experimental conditions, subjects performed practice trials until they felt at ease with the recording apparatus (typically, $10-15$ trials). Experimental trials were divided into three separate runs (one for each condition). The first plane tested was counterbalanced across individuals.

Data analysis. The dependent variables of interest were as follows: (1) the signed error, which consists of the average deviation (in degrees) of the response from the target location and was taken to indicate potential directional response biases (e.g., a tendency to present a leftward or rightward shift in the horizontal plane); and (2) the root mean square (RMS) error, which represents the SD for each subject of the differences between target locations and the localization response, and was taken as a measure of overall precision. For each condition/run, the localization error was computed in only one dimension (either horizontal or vertical), in accordance with the auditory plane being tested. Single trials with signed errors that were $>3$ SDs above the mean deviation per target location per group were considered outliers and were removed from all further analyses. As such, $0.44 \%$ of the total number of trials for the blind were excluded, whereas $0.96 \%$ of the trials were removed for the sighted.

We divided the blind subjects a posteriori using the binomial test into two groups based on their performance in the monaural horizontal condition; that is, on the proportion of trials in which they pointed to the correct side when the sound originated from the occluded side. The blind subjects whose performance was significantly $(p \leq 0.01)$ above chance were assigned to the EB "superior performance" (EBSP) group, whereas the rest were assigned to the EB "normal performance" (EBNP), in accordance with the procedure outlined by Doucet et al. (2005). As a result of this procedure, six early blind subjects were assigned to the EBSP group and five were assigned to the EBNP group. The EBSP subjects on average pointed to the correct hemifield on $80 \%$ of the trials (range, $65-92 \%)$, whereas the EBNP subjects pointed correctly on $34 \%$ of the trials (range, $0-53 \%$ ). In comparison, the sighted pointed correctly on $39 \%$ of the trials (range, $0-92 \%$ ).

For data analyses purposes, the 25 loudspeakers in the horizontal plane were divided into four equal portions (omitting the data collected from the central speaker, $0^{\circ}$ ), each containing 6 loudspeakers. The data from each speaker within a portion were averaged for each subject, thus creating a LOCATION variable with four levels (far-left, left-center, right-center, and far-right). A similar procedure was performed for the vertical plane loudspeakers, with the 18 loudspeakers being divided into three equal portions of six loudspeakers (top, central, bottom). The resulting data were then entered into three separate GROUP (three) $\times$ LOCATION (four, horizontal plane; three, vertical plane) repeated-measures ANOVAs (one per condition). 


\section{A}
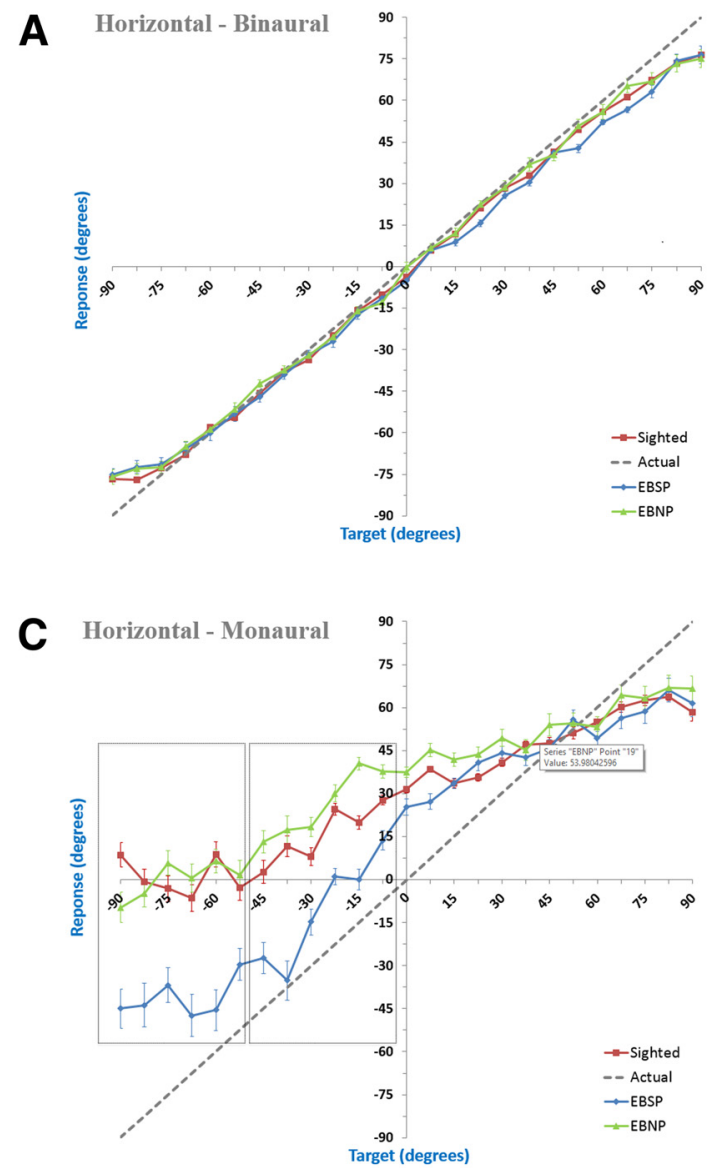

\section{E}

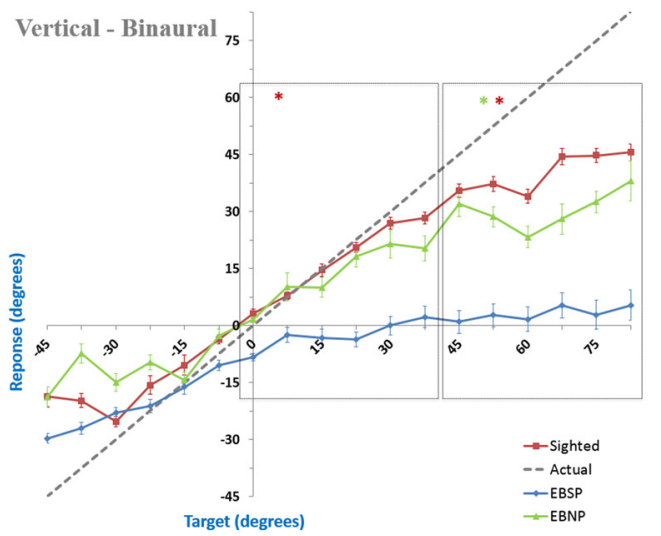

B

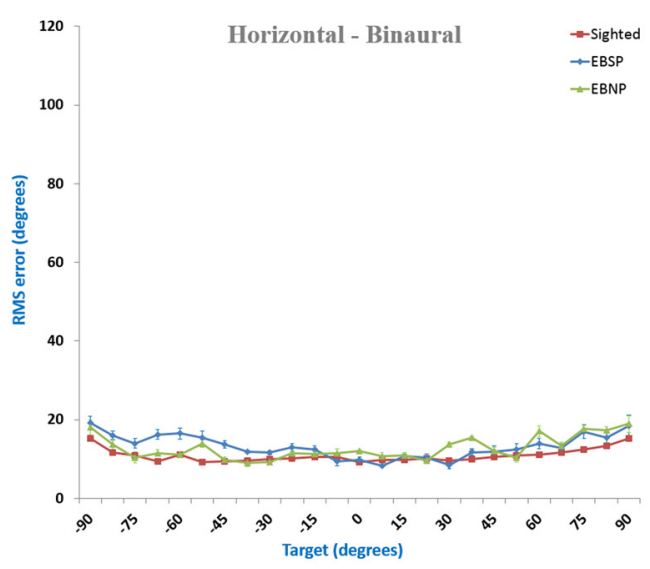

D

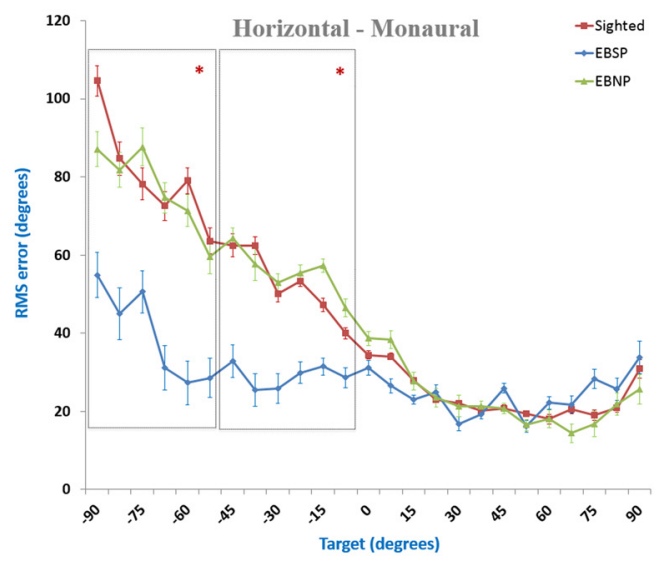

$\mathbf{F}$

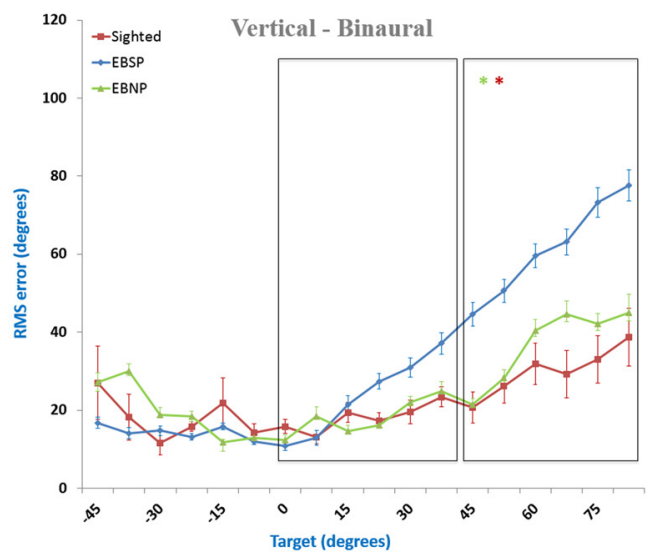

Figure 1. Sound localization in the horizontal and vertical planes. $A, C, E$, The left graphs illustrate the average group responses as a function of target position (and serve to illustrate the signed error relative the ideal performance, as indicated by the dashed lines) for the binaural horizontal task $(\boldsymbol{A})$, and the monaural horizontal task $(\boldsymbol{C})$ and vertical task $(\boldsymbol{E})$. Note that for the monaural condition, negative angles on the abscissa correspond to the obstructed ear side, while positive angles correspond to the unobstructed ear side. $\boldsymbol{B}, \boldsymbol{D}, \boldsymbol{F}$, The right graphs illustrate the average group RMS error as a function of target position, again for the binaural horizontal task $(\boldsymbol{B})$, and the monaural horizontal task $(\boldsymbol{D})$ and vertical task $(\boldsymbol{F})$. For all graphs, the error bars represent the SE, and the dotted rectangles indicate a portion of space where significant group differences were observed for either the signed or RMS error. A red asterisk corresponds to a significant difference between the EBSP group and the sighted group, whereas a green asterisk corresponds to a significant difference between the EBSP and EBNP groups.

\section{Results}

\section{Horizontal-binaural condition}

RMS ERROR: There was a statistical trend for the LOCATION variable $(F=3.10, p=0.07)$, while The GROUP variable $(F=1.90, p=$ $0.17)$ and the SPACE $\times$ LOCATION interaction were nonsignificant $(F=0.45, p=0.72$, Fig. $1 A)$. SIGNED ERROR: There was a significant effect of LOCATION $(F=28.45, p<0.001)$ where performance was significantly different among all the quadrants (all $p<$ 0.004 ) with the exception of the two central quadrants, where per- formance did not differ $(p=0.27)$. The GROUP variable $(F=0.231$, $p=0.11)$ and the SPACE $\times$ LOCATION interaction were nonsignificant $(F=0.40, p=0.73)$. In other words, the subjects' accuracy decreased as a function of eccentricity due to a bias to point toward the midline side of the target (Fig. 1B).

\section{Horizontal-monaural condition}

RMS ERROR: There was a highly significant effect of LOCATION $(F=26.05, p<0.001)$ resulting from an overall better 
performance for the unoccluded side than for the occluded side (Fig. 1C). Direct comparisons between the two blind subgroups were not considered here to avoid circularity, given that the group assignment was performed on the basis of their general ability to localize sounds monaurally. However, the group definition was independent of performance by the control subjects, and since we had hypothesized that the EBSP would be better than the performance for the sighted in this task, we proceeded to examine the simple effects. Indeed, the EBSP subjects were found to be significantly better than the sighted subjects in both the far-occluded quadrant $(p=0.04)$ and the center-occluded quadrant $(p=0.02)$. In comparison, the EBNP subjects were not statistically different from sighted subjects in all quadrants (all $p>0.7$ ). SIGNED ERROR: Similarly, there was a highly significant effect of LOCATION $(F=28.55, p<0.001)$. The signed error was also smaller for the EBSP subjects than for the sighted subjects in both the far-occluded quadrant and the centeroccluded quadrant, though these effects did not quite reach the statistical criteria for significance $(p=0.07$ and $p=0.06$, respectively; Fig. 1D).

\section{Vertical condition}

RMS ERROR: The effects of LOCATION $(F=23.73, p<0.001)$, GROUP $(F=3.58, p=0.04)$, and LOCATION $\times$ GROUP interaction $(F=4.19, p=0.02)$ were all found to be statistically significant (Fig. 1E). Analysis of the simple effects revealed that the EBSP group was worse than both the EBNP $(p=0.04)$ and the sighted $(p=0.003)$ groups in the top portion $\left(+45^{\circ}\right.$ to $\left.+82.5^{\circ}\right)$ of the vertical plane. No significant group differences were observed for the central and bottom portions (all $p>0.2$ ). SIGNED ERROR: The effects of LOCATION $(F=83.71, p<0.001)$, GROUP $(F=3.75$, $p=0.04)$, and LOCATION $\times$ GROUP interaction $(F=3.41, p=$ 0.04 ) were all found to be statistically significant (Fig. $1 f$ ). Analysis of the simple effects revealed that the EBSP group was worse than both the EBNP $(p=0.05)$ and the sighted $(p=0.002)$ groups in the top portion $\left(+45^{\circ}\right.$ to $\left.+82.5^{\circ}\right)$ of the vertical plane. The EBSP group was also worse than the sighted group $(p=0.02)$ in the central portion of the speaker layout; they were also worse than the EBNP group here, though the comparison failed to reach significance $(p=0.08)$. To summarize, the EBSP group displayed lower accuracy in the top portion of the vertical plane as a result of their tendency to considerably undershoot the target locations.

Finally, to obtain a metric of how blind individuals' performance in the vertical condition relates to their performance in the horizontal-monaural condition that is independent of our group assignment, we correlated RMS error scores from both conditions on an individual basis. Specifically, we first computed measures of the average unsigned error within the regions of space where group differences were observed [horizontal: $-2.5^{\circ}$ to $-45^{\circ}$ (center-occluded quadrant) and $-52.2^{\circ}$ to $-90^{\circ}$ (faroccluded quadrant); vertical: from $+45^{\circ}$ to $+82.5^{\circ}$ ] and then proceeded to correlate the measures between planes. In good agreement with the group contrasts, we found that the measures were negatively correlated with one another in the center-occluded quadrant ( $r=-0.62 ; p=0.04$ ) for the blind individuals (Fig. 2). This finding strongly contrasts with the nonsignificant positive correlation observed in the sighted $(r=0.32 ; p=0.32)$, as evidenced by the significant difference between the two correlation coefficients $(z=-2.21 ; p=0.03)$. The correlations were slightly weaker for the far-occluded quadrant where performance was generally poorer for the both the blind subjects $(r=-0.40 ; p=$ $0.23)$ and the sighted subjects $(r=0.34 ; p=0.28)$.

\section{Discussion}

To investigate the potential existence of trade-offs in the development of enhanced perceptual abilities in the early blind, and to address the long-standing discrepancy regarding their spatial hearing abilities, we asked both blind and sighted individuals to perform sound localization tasks under three distinct listening conditions. The results in the binaural horizontal task are highly consistent with previous findings showing equal performance between blind and sighted individuals in the horizontal plane (Lessard et al., 1998; Gougoux et al., 2005). The findings of the monaural task are again highly consistent with previously published findings showing a clear dichotomy within the results for the early blind, where half of the subjects perform in a manner similar to the sighted, whereas the other half significantly outperform them (Lessard et al., 1998; Doucet et al., 2005; Gougoux et al., 2005). Lastly, the finding that some blind subjects are worse than sighted subjects when localizing in the vertical plane is also consistent with previous reports (Zwiers et al., 2001; Lewald, 2002). However, it was not clear from prior studies whether there was a relationship across both tasks, or whether separate populations of blind persons were being tested. Here, we provide the first data on both vertical and monaural horizontal sound localization in the same group of subjects, and we clearly show that the early blind individuals who best perform the monaural horizontal localization task (EBSP group) are largely the same individuals who perform worse in the vertical condition. Moreover, there is a negative correlation between the accuracy on the two tasks when taking all blind individuals together. These results argue against the idea that early blind individuals have generalized perceptual enhancements in their remaining sensory modalities (as is also supported by the recent findings of Gori et al., 2014); rather, they suggest the existence of a trade-off in the localization proficiency of early blind individuals between the two auditory spatial planes.

The finding that a trade-off takes place across individuals is surprising, because the ability to localize in the vertical plane is believed to rest on the use of spectral cues (Middlebrooks and Green, 1991; Blauert, 1997), whereas the accurate monaural localization of the EBSP is also believed to rest at least in large part on the use of spectral cues, as well evidenced by studies manipulating spectral content during monaural localization (Doucet et al., 2005; Voss et al., 2011), and others showing enhanced performance in peripheral auditory space where spectral cues are helpful in resolving spatial ambiguities (Röder et al., 1999; Voss et al., 2004). One potential explanation for the apparent discrepancy relates to the type of spectral information being used in each specific context. Candidate spectral features for localization include overt peaks, notches, steep gradients, and covert peaks (Carlile et al., 2003). The presence of a prominent spectral notch in HRTFs that could provide a reliable cue to source elevation has been noted by several authors (Hebrank and Wright, 1974; Bloom, 1977; Butler and Belendiuk, 1977), as the center frequency of this notch has been shown to increase systematically from $\sim 5$ to $14 \mathrm{kHz}$ with increases in source elevation. Relatedly, Macpherson (1997) has suggested that in fact it is the entire spectrum (and not just the notch) that is compared with stored templates for different elevation positions. In contrast, it has been suggested that monaural localization primarily rests on the analysis of covert spectral features (i.e., that become evident when HRTFs are compared across locations). In other words, the covert peak indicates the spatial location at which a particular frequency is maximally transmitted to the ear drum (Musicant and Butler, 1984; Rogers and Butler, 1992). Interestingly, covert peak 
A

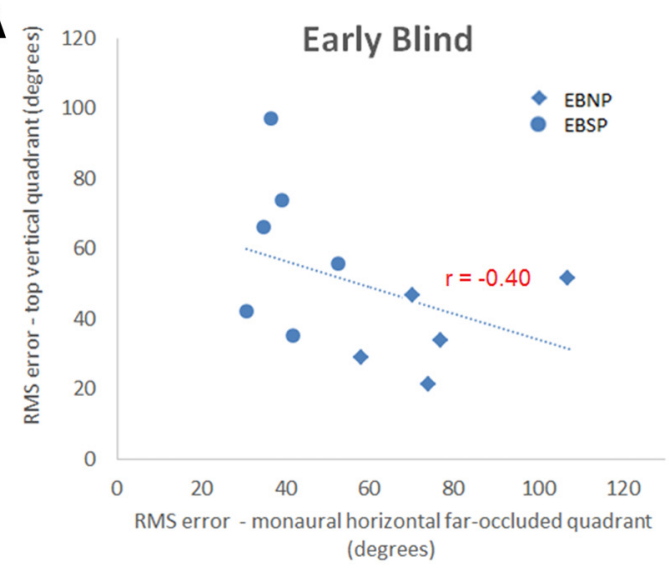

B

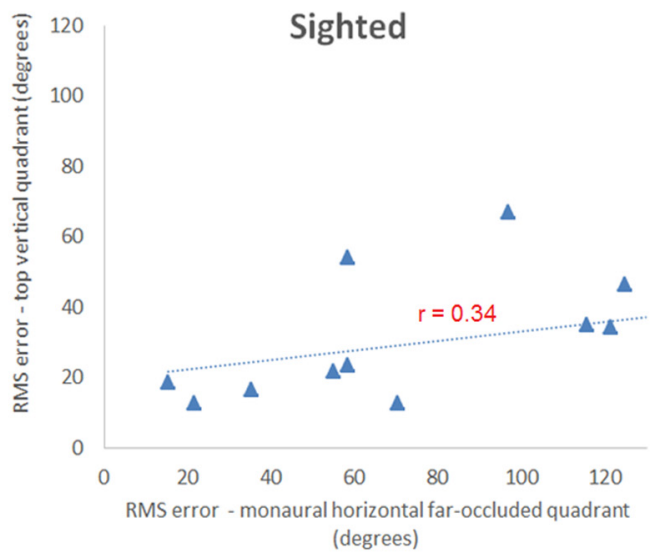

C

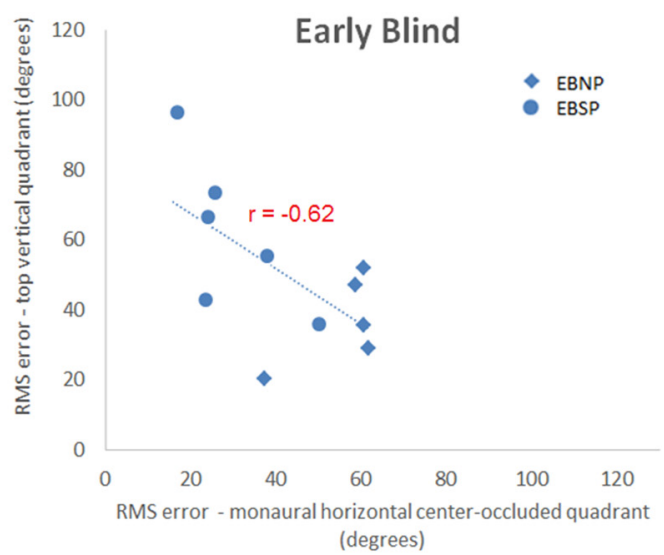

D

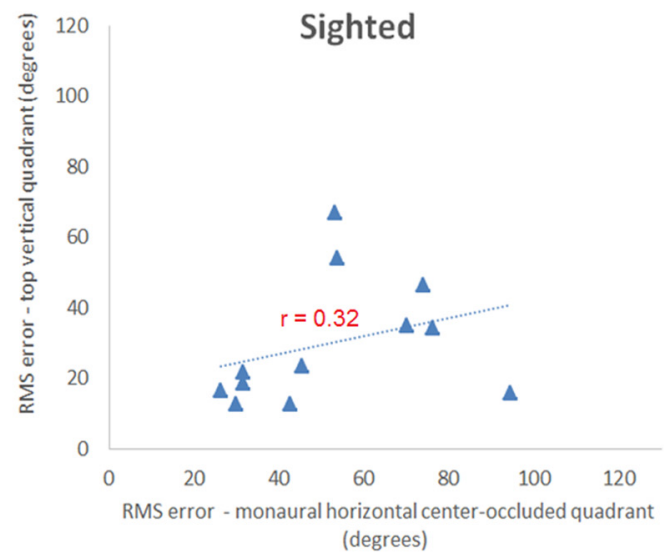

Figure 2. Correlation between the performance in the monaural horizontal and vertical tasks. $\boldsymbol{A}, \boldsymbol{B}$, Correlation for the horizontal positions in the far-occluded quadrant for the early blind and sighted groups, respectively. C, D, Correlation for the horizontal positions in the center-occluded quadrant for the early blind and sighted groups, respectively. Each data point represents the RMS error within the portions of space for each task. The dotted lines represent the linear trend for each group.

analysis has also been suggested to help resolve locations on the cone of confusion-where interaural time and level difference cues are insufficient to resolve whether a sound source lies in front or behind the axis (Musicant and Butler, 1984; Humanski and Butler, 1988)—which is located in peripheral auditory space, whereas the blind have previously shown higher spatial resolution in the horizontal plane (Röder et al., 1999; Voss et al., 2004; Després et al., 2005). Consequently, if the early blind do indeed process these cues more efficiently, this could explain their superiority in monaural listening conditions and in the auditory periphery when localizing with both ears. The corollary of this assumption is that in turn the early blind who learn to efficiently process these cues, are poorer at processing the specific notches/ spectra associated with localization in elevation.

An alternative, though not mutually exclusive, explanation relates to a change in the mapping or the weighting of the spectral cues between planes. Indeed, it is well established that spectral cues for elevation can be learned based on environmental feedback (Hofman et al., 1998), and that the pinna-induced spectral shape cues are changing during life because the ears keep growing, and that listeners adapt to these changing cues (Otte et al., 2013). The present data suggest that learning one set of mappings between spectral acoustic cues and spatial positions might preclude a different mapping, where one can use the cues effectively for one or the other auditory plane, but not both. While sighted individuals and some blind persons (those in the EBNP group) learn this mapping primarily for the vertical dimension, where binaural cues provide limited spatial information, some early blind individuals instead seem to learn this mapping for the horizontal plane, which may then make the cues unavailable for the vertical plane. This change in mapping may stem from the greater ecological benefit of being accurate in the horizontal plane as opposed to the vertical one. Indeed, when navigating in outdoor environments, the majority of navigation-relevant sound sources are located in the horizontal plane.

Last, while the fact that the EBSP may also make better use of intensity (level) cues to localize sounds monaurally in the horizontal plane remains a possibility, the aforementioned studies that specifically investigated the role played by spectral cues (Doucet et al., 2005; Voss et al., 2011) suggest that it is unlikely. Furthermore, there is ample evidence of early blind individuals showing an enhanced ability to detect fine spectral changes in nonspatial tasks (Gougoux et al., 2005; Voss and Zatorre, 2012), whereas there is little to no such evidence for the discrimination of fine intensity changes (which are of course the basis of headshadow cues) (Starlinger and Niemeyer, 1981; Voss and Zatorre, 2012).

To conclude, here we present findings that address a longstanding discrepancy in the literature concerning the sound localization abilities of the blind. We show that the blind individuals who have the highest accuracy at localizing sounds monaurally in the horizontal plane (EBSP group) are also the ones who show the greater deficit relative to sighted individuals when localizing in the vertical plane. This finding argues against 
the notion that early blind individuals have a generalized spatial hearing enhancement, and suggests instead the existence of a trade-off in their localization proficiency, where developing exceptional abilities in the horizontal plane comes at the expense of localization abilities in the vertical plane.

\section{References}

Blauert J (1997) Spatial hearing. The psychophysics of human sound localization. Cambridge, MA: MIT.

Bloom, (1977) Creating source elevation illusions by spectral manipulation. J Audio Eng Soc 25:560-565.

Butler RA, Belendiuk K (1977) Spectral cues utilized in the localization of sound in the median sagittal plane. J Acoust Soc Am 61:1264-1269. Medline

Carlile S, Martin R, McAnally K (2003) Spectral information in sound localization. Int Rev Neurobiol 70:399-434. CrossRef Medline

Després O, Candas V, Dufour A (2005) The extent of visual deficit and auditory spatial compensation: evidence from self-positioning from auditory cues. Brain Res Cogn Brain Res 23:444-447. CrossRef Medline

Doucet ME, Guillemot JP, Lassonde M, Gagné JP, Leclerc C, Lepore F (2005) Blind subjects process auditory spectral cues more efficiently than sighted people. Exp Brain Res 160:194-202. CrossRef Medline

Gori M, Sandini G, Martinoli C, Burr DC (2014) Impairment of auditory spatial localization in congenitally blind human subjects. Brain 137:288293. CrossRef Medline

Gougoux F, Zatorre RJ, Lassonde M, Voss P, Lepore F (2005) A functional neuroimaging study of sound localization: visual cortex activity predicts performance in early-blind individuals. PLoS Biol 3:e27. CrossRef Medline

Hebrank J, Wright D (1974) Spectral cues used in the localization of sound sources on the median plane. J Acoust Soc Am 56:1829-1834. CrossRef Medline

Hofman PM, Van Riswick JG, Van Opstal AJ (1998) Relearning sound localization with new ears. Nat Neurosci 1:417-421. CrossRef Medline

Humanski RA, Butler RA (1988) The contribution of near and far ear toward of sound in the sagittal plane. J Acoust Soc Am 83:2300-2310. CrossRef Medline

Lessard N, Paré M, Lepore F, Lassonde M (1998) Early-blind human subjects localize sound sources better than sighted subjects. Nature 395:278280. CrossRef Medline

Lewald J (2002) Vertical sound localization in blind humans. Neuropsychologia 40:1868-1872. CrossRef Medline
Lewald J (2007) More accurate sound localization induced by short-term light deprivation. Neuropsychologia 45:1215-1222. CrossRef Medline

Macpherson EA (1997) A comparison of spectral correlation and local feature-matching models of pinna cue processing. J Acoust Soc Am 101:3104A

Middlebrooks JC, Green DM (1991) Sound localization by human listeners. Annu Rev Psychol 42:135-159. CrossRef Medline

Musicant AD, Butler RA (1984) The psychophysical basis of monaural localization. Hear Res 14:185-190. CrossRef Medline

Otte RJ, Agterberg MJ, Van Wanrooij MM, Snik AF, Van Opstal AJ (2013) Age-related hearing loss and ear morphology affect vertical but not horizontal sound-localization performance. J Assoc Res Otolaryngol 14:261273. CrossRef Medline

Röder B, Teder-Sälejärvi W, Sterr A, Rösler F, Hillyard SA, Neville HJ (1999) Improved auditory spatial tuning in blind humans. Nature 400:162-166. CrossRef Medline

Rogers ME, Butler RA (1992) The linkage between stimulus frequency and covert peak areas as it relates to monaural localization. Percept Psychophys 52:536-546. CrossRef Medline

Starlinger I, Niemeyer W (1981) Do the blind hear better? Investigations on auditory processing in congenital or early acquired blindness. I. Peripheral functions. Audiology 20:503-509. CrossRef Medline

Tabry V, Zatorre RJ, Voss P (2013) The effect of blindfolding and of pointing method on sound localization. Front Aud Cogn Neurosci 4:932.

Van Wanrooij MM, Van Opstal AJ (2004) Contribution of head shadow and pinna cues to chronic monaural sound localization. J Neurosci 24: 4163-4171. CrossRef Medline

Voss P, Zatorre RJ (2012) Occipital cortical thickness predicts performance on pitch and musical tasks in blind individuals. Cereb Cortex 22:24552465. CrossRef Medline

Voss P, Lassonde M, Gougoux F, Fortin M, Guillemot JP, Lepore F (2004) Early- and late-onset blind individuals show supra-normal auditory abilities in far space. Curr Biol 14:1734-1738. CrossRef Medline

Voss P, Gougoux F, Zatorre RJ, Lassonde M, Lepore F (2008) Differential occipital responses in early- and late-blind individuals during a soundsource discrimination task. Neuroimage 40:746-758. CrossRef Medline

Voss P, Lepore F, Gougoux F, Zatorre RJ (2011) Relevance of spectral cues for auditory spatial processing in the occipital cortex of the blind. Front Psychol 2:48. CrossRef Medline

Zwiers MP, Van Opstal AJ, Cruysberg JR (2001) A spatial hearing deficit in early blind individuals. J Neurosci 21:RC142. Medline 